\title{
Germanica
}

les pays germanophones de 1880 à nos jours

\section{Edouard Jolly, Günther Anders. Une politique de la}

\section{technique}

Paris, Michalon, 2017, 120 p.

\section{Martine Benoit}

\section{OpenEdition}

\section{Journals}

Édition électronique

URL : http://journals.openedition.org/germanica/5089

DOI : $10.4000 /$ germanica. 5089

ISSN : 2107-0784

Éditeur

Université de Lille

\section{Édition imprimée}

Date de publication : 31 juillet 2018

Pagination : 184-186

ISBN : 9782913857414

ISSN : 0984-2632

Référence électronique

Martine Benoit, « Edouard Jolly, Günther Anders. Une politique de la technique », Germanica [En ligne], 62 2018, mis en ligne le 31 juillet 2018, consulté le 16 janvier 2021. URL : http://journals.openedition.org/ germanica/5089; DOI : https://doi.org/10.4000/germanica.5089 


\section{Comptes rendus de lecture}

Florence Baillet (dir.), Einar Schleef par-delà le théâtre. Mise en scène, écriture, peinture, photographie, Rennes, Presses Universitaires de Rennes, 2016, coll. «Le Spectaculaire », 204 p.

L'ouvrage collectif dirigé par Florence Baillet est le fruit d'un colloque international organisé par l'équipe de recherche en études germaniques de l'université Paris 3 Sorbonne Nouvelle (le CEREG), en collaboration avec l'institut de littérature allemande de l'université Humboldt de Berlin et l'institut de recherches en études théâtrales de l'université Paris 3 Sorbonne Nouvelle. Il s'est tenu dans différents lieux culturels (le Goethe-Institut de Paris, le théâtre national de La Colline) et universitaire (Université Paris 3 Sorbonne Nouvelle) en novembre 2014.

Centré sur la figure de l'artiste allemand Einar Schleef (1944-2001), le présent recueil éclaire tous les pans de son œuvre protéiforme, englobant photographie et peinture, mise en scène et écriture, comme l'indique le sous-titre. C'est bien cet intérêt porté sur toutes les formes pratiquées par Einar Schleef, et donc en quelque sorte sur le geste artistique au fondement de cette œuvre, qui constitue la particularité de l'ouvrage. Cette approche lui permet de relever deux défis : le livre se présente à la fois comme une introduction à destination d'un public francophone qui connaît très peu l'œuvre de cet artiste (on recense à ce jour une seule thèse soutenue en France portant sur Einar Schleef ${ }^{1}$ ), et comme un nouveau jalon dans la recherche internationale. En effet, la problématique de la matérialité qui sous-tend l'ouvrage et apparaît comme

1. - Charlotte Bomy, Théâtralité et intermédialité dans les spectacles de Frank Castorf, Einar Schleef et Heiner Goebbels, thèse de doctorat soutenue en 2008 à l'université Marc Bloch de Strasbourg/ Freie Universität Berlin. 
un concept fondamental pour comprendre la démarche artistique de Schleef est en même temps une question inédite qui permet d'articuler autrement différents éléments de l'esthétique schleefienne.

Né en 1944 à Sangerhausen en Thuringe, Einar Schleef grandit en RDA puis passa à l'Ouest en 1976, peu avant l'affaire Biermann². La subversion et le scandale semblent rythmer sa trajectoire. Inscrit d'abord dans la section peinture de l'école des Beaux-Arts de Weißensee à Berlin, il en fut exclu pour indiscipline avant de la réintégrer en photographie et scénographie (p. 13). Alors qu'il travailla avec les plus grands, B.K. Tragelehn, Heiner Müller ou Elfriede Jelinek, à l'Est comme à l'Ouest, de Berlin-Est à Vienne en passant par Francfort, du Berliner Ensemble au Burgtheater, nombre de ses mises en scène suscitèrent le rejet. Dans sa très éclairante introduction, Florence Baillet décrit « une œuvre à part, singulière et radicale, refusant tout compromis. » (p. 15). De la lecture de l'introduction et des articles se dégage ainsi la conviction que la provocation n'est pas l'objectif premier de l'artiste mais l'effet produit par l'investissement éthique du champ artistique guidé par un intérêt profond pour la marge, « les oubliés et les sacrifiés de l'histoire » (p. 10). Les éléments devenus caractéristiques de son œuvre, comme l'accent mis sur le corps, le conflit, le refus de toute psychologisation, le retour au chœur et la référence à la tragédie antique, la mise en valeur des rôles féminins et l'abandon de la perspective centrale, apparaissent dès lors comme faisant partie intégrante d'un projet politique. Le prisme de la matérialité, " terme exclu de l'économie occidentale de la représentation » (p. 19), qui met en avant l'événement et le matériau, au détriment de la référentialité, le médium même, au détriment de «l'ordre symbolique » (p. 19), permet précisément de saisir la dimension éthique au cœur des caractéristiques et usages de formes au premier abord disparates. Dans le même temps, la question du matériau place le geste schleefien dans le sillage d'esthétiques modernes et contemporaines qu'il radicalise. Ainsi son œuvre, que parcourent par ailleurs les références à Nietzsche, a-t-elle pu être étudiée sous l'angle plus large du théâtre postdramatique (Hans-Thies Lehmann).

$\mathrm{Au}$ caractère novateur d'un ouvrage scientifique faisant progresser l'état des connaissances sur Einar Schleef, le volume allie la vocation quasi pédagogique d'un transfert culturel, jouant le rôle de passeur d'un objet culturel de l'aire germanique à l'aire francophone. Les douze contributions que rassemble le livre sont en français. Parmi elles, quatre sont

2. - C'est-à-dire avant que le poète et chansonnier est-allemand Wolf Biermann ne soit destitué de sa citoyenneté est-allemande après un concert à Cologne à l'automne 1976, ce qui entraîna la signature et la diffusion d'une lettre de protestation de nombreux artistes est-allemands, à laquelle le pouvoir répondit par une politique répressive, entraînant à son tour une vague d'émigration d'artistes. 
traduites de l'allemand : par Florence Baillet (le prologue à deux voix d'Helene Varopoulou et Hans-Thies Lehmann), Charlotte Bomy (l'article de Miriam Dreysse et celui de Christina Schmidt) et Sarah Neelsen (le texte de Kristin Schulz). La bibliographie sélective en fin d'ouvrage rend compte de manière quasi exhaustive de la littérature secondaire publiée en français, comme le mentionne la notice introductive (p. 193). La « chronologie des principaux spectacles d'Einar Schleef » établie par Charlotte Bomy (p. 191-192), dans laquelle les titres de toutes les pièces sont traduits en français, fournit un instrument précieux de repérage dans l'œuvre du metteur en scène, à la portée d'un lectorat non initié. Il est utilement complété par un index des noms propres et un index des titres des œuvres et spectacles de Schleef. Enfin, la figure du passeur entre les aires culturelles par excellence qu'est le traducteur est mise en valeur dans l'entretien qu'a mené Florence Baillet avec Heinz Schwarzinger (connu aussi sous le pseudonyme « Henri Christophe »), qui a traduit deux pièces de Schleef en français (Trompettes de la mort et Trilogie Nietzsche) ${ }^{3}$. Les images en noir et blanc qui accompagnent plusieurs articles, ainsi que le cahier central de vingt-quatre planches contenant des reproductions en couleur des dessins, croquis, brouillons et tableaux de l'artiste, ainsi que des photographies en noir et blanc de Schleef ou de ses mises en scène, parachèvent la dynamique de transmission qui innerve le recueil.

Malgré la multiplicité des interventions, le recueil est également d'une remarquable cohérence. Au bas des pages, les références aux autres articles du volume resserrent continûment les liens entre les textes. Organisé en trois temps, le livre s'articule autour de la dimension politique du théâtre de Schleef et de son essai Droge Faust Parsifal (1977), puis des strates que constitue dans le texte l'intrication du matériau de l'histoire individuelle et collective, et enfin d'un troisième volet transcendant en quelque sorte les formes artistiques et rapprochant le théâtre des arts visuels ou sonores. L'ouvrage rend justice à la diversité de l'œuvre schleefienne. Y sont abordés le monumental roman qu'il consacra à sa mère, Gertrud, publié en deux parties en 1980 et 1984 (Sarah Neelsen), ses photographies (Sylvie Arlaud), ainsi que la peinture (Marielle Silhouette) et la scénographie (Christina Schmidt), ou encore les brouillons conservés aux archives (Kristin Schulz), bien que le théâtre y occupe une place prépondérante (Emmanuel Béhague consacre notamment un article dense à Wezel, une pièce peu connue de Schleef) et que certains articles de la première partie, consacrés au rôle

3. - Un recueil de nouvelles, Désordre, et un texte d'Einar Schleef (accompagné d'un CD) intitulé Gertrud: Monologue pour chœur de femmes ont en outre été traduits par Marie-Luce Bonfanti et Crista Mittelsteiner pour les éditions Le Ver à soie, respectivement en 2014 et 2016. 
de la femme et du chœur, semblent quelque peu redondants. Parmi ses pièces, la mise en scène en 1998 d'Une pièce de sport (Ein Sportstück) d'Elfriede Jelinek, qui lui apporta la reconnaissance internationale, est sans conteste celle qui trouve le plus d'écho dans le présent ouvrage (la contribution d'Aline Vennemann est à cet égard emblématique). Ce dernier bénéficie de cet agencement choral et somme toute assez féminin, qu'un Schleef ne désavouerait pas, qui fait travailler ensemble spécialistes, de part et d'autre du Rhin, de l'œuvre d'Einar Schleef et de celle d'Elfriede Jelinek ou encore de Heiner Müller, chercheurs reconnus et jeunes chercheurs dans le domaine du théâtre contemporain de langue allemande.

La variété des formes textuelles (introduction, prologue, entretien, articles), la richesse et la qualité des matériaux mis à disposition par ce volume compact en font un ouvrage à la fois accessible et incontournable sur Einar Schleef.

Bénédicte Terrisse

Edouard Jolly, Günther Anders. Une politique de la technique, Paris, Michalon, 2017, 120 p.

En 1983, Günther Anders recevait le prix Theodor W. Adorno de la ville de Francfort-sur-le-Main'1. C'est à Günter Kunert qu'il revient l'honneur de tenir la laudatio - Kunert qui, dès 1965 et alors qu'il était encore citoyen de RDA, publiait Der ungebetene Gast (L'hôte indésirable), un recueil de poèmes dans lequel il écrivait vouloir mettre en garde contre la « déshumanisation, la perte, possible et irrémédiable, de la conditio humana $»^{2}$ : dans ce recueil, le poème «Der Schatten » («L'ombre ») est directement inspiré de Günther Anders. En effet, dans son ouvrage L'Homme sur le pont. Journal d'Hiroshima et de Nagasaki (1959), Günther Anders citait le discours de clôture du « 4e Congrès international contre la bombe atomique et la bombe à hydrogène et pour le désarmement » tenu à Tokyo le 20 août 1958 où l'orateur affirmait : "Sur l'un des ponts d'Hiroshima se tient un homme qui chante et joue de la guitare. Regardez-le. Là où vous attendez de voir son visage, vous ne trouverez aucun visage mais un rideau : car il n'a plus de visage. Et là où vous attendez sa main, vous ne trouverez pas de main mais une pince en acier ». Pour Günther Anders, l'homme sur le pont est « la dernière image du dernier homme »; dans le poème « Der Schatten », cet homme symbolise l'extermination totale de toute vie humaine - cependant, le

1. - Prix remis tous les 3 ans, le dernier lauréat a été en 2015 Georges DidiHuberman. On trouvera ici les noms des 14 lauréats à ce jour : https://www.frankfurt. de/sixcms/detail.php?id=8650\&_ffmpar[_id_inhalt] $=21490$.

2. - Günter Kunert, Der ungebetene Gast, Gedichte, Berlin und Weimar, AufbauVerlag, 1965. 
dernier vers du poème de Kunert autorisait encore un espoir : la plaie béante que représente cette ombre pour l'humanité est encore susceptible de se refermer. En 1983, dans sa laudatio, Kunert se fait plus rude, nous sommes, écrit-il, « au bord du gouffre », il intitule son discours : «Réflexions inquiètes et inquiétantes ».

Le petit livre d'Édouard Jolly est une excellente manière d'entrer dans la pensée de Günther Anders. Actuellement chercheur à l'IRSEM (Institut de recherche stratégique de l'école militaire) et chercheur associé au laboratoire STL (Savoirs, Textes, Langages) de l'Université de Lille, Édouard Jolly nous livre ici un premier aperçu de sa thèse, soutenue en 2013 sous le titre : «Les Ombres du monde. Günther Anders et le refus du nihilisme ». Ce premier livre (car on peut espérer que la thèse d'Édouard Jolly paraisse rapidement) se concentre sur la relation entre technique et politique. La pensée politique des écrits d'après-guerre de Günther Anders nous donne en effet d'abord à réfléchir aux bouleversements écologiques et politiques du XXI ${ }^{\mathrm{e}}$ siècle, avec comme point central, l'angoissante perspective d'une espèce humaine qui risque, voire qui est en train de produire son extinction à l'aide de son propre fait. Edouard Jolly nous emmène dans sa lecture attentive et savante de Die Antiquiertheit des Menschen (publié en deux tomes, en 1956 et 1980, traduit en français sous le titre L'obsolescence de l'homme), des écrits sur Hiroshima (Hiroshima ist überall - Hiroshima est partout, paru en 1982), des Zehn Thesen zu Tschernobyl (Dix thèses sur Tchernobyl, publié en 1986) et nous propose un développement en quatre grandes étapes : comment penser la technique moderne ; que signifie produire dans un monde déshumanisé ; comment comprendre la notion d'obsolescence de l'homme lui-même ; pourquoi une politique de la technique est-elle déterminante face aux défis écologiques qu'Anders voyait s'annoncer pour le XXI ${ }^{\mathrm{e}}$ siècle. Édouard Jolly nous fait entrer avec clarté et méthode dans la pensée de Günther Anders et les différentes notions centrales de sa philosophie. La première partie se concentre sur la notion de « décalage prométhéen », décalage entre les possibilités techniques et l'homme, entre les capacités de produire et celles de représenter - entre la réalisation d'une action et ses conséquences : on ne peut parler de technophobie chez Anders, Édouard Jolly propose le terme de « conservatisme », à savoir : " préserver l'étendue la plus large des possibilités d'expérience en faveur d'une autonomie individuelle » (p. 26). Autre notion centrale : le «totalitarisme des appareils », à savoir l'exclusion de l'homme de tout processus de production et finalement de tout monde, la perte de contrôle après la maîtrise : "L'homme en tant qu'être vivant biologique, éprouve ses limites physiques dans l'adaptation requise par ses appareils » (p. 40) - et les hommes, devenus inadaptés, deviennent, selon l'expression de Günther Anders, « des hommes sans monde » (citation p. 55). Troisième étape de la pensée de Günther Anders : la 
notion d'obsolescence de l'homme, ce processus de dépossession qui tend à exclure l'homme du monde : ce qui caractérise l'homme de notre société moderne, c'est son conformisme, sa soumission, l'adaptation passive, l'obéissance généralisée (des passages qui donnent envie de se replonger dans La Boétie et son Discours de la servitude volontaire). Édouard Jolly finit cette introduction savante et d'une écriture limpide par une analyse des Dix thèses après Tchernobyl, texte qui permet de poser les fondements d'une anticipation propre à la menace nucléaire, une menace "dont l'échelle spatio-temporelle dépasse celle de toute civilisation historique » (p. 90) : dans ce texte riche et angoissant, Anders affirme entre autres la nécessité de la panique et des affects moraux pour faire face à la réalité de la situation atomique. Comme le résume Édouard Jolly : « Nous serions les témoins du "temps de la fin", d'une époque historique dont la finalité des finalités nous met dans l'impossibilité logique de parler au futur antérieur : une fois la dévastation atomique déclenchée, une fois l'extinction de l'espèce humaine, il n'y aura plus personne pour raconter l'événement. Nous ne pouvons ni nous projeter après l'apocalypse, ni anticiper un monde où la vie ne trouverait aucun environnement adéquat et où plus aucune histoire ne serait à raconter » (p. 91).

Dans une interview donnée en 1990, Günter Kunert revenait sur les reproches réitérés concernant son pessimisme légendaire et répliquait : « Je me considère comme un observateur lucide qui, contrairement aux autres, ne cherche pas à refouler ce qui nous attend $»^{3}$. On pourrait en dire tout autant de Günther Anders.

Martine Benoit

3. - Interview parue dans Dissidenten ? Texte und Dokumente zur DDR-'Exil' Literatur, Berlin, Volk-und-Wissen-Verlag, 1990. 